\title{
Correction to: Advanced Concepts in Quantum Field Theory
}

\section{Correction to:}

J. M. Cline, Advanced Concepts in Quantum, Field Theory, SpringerBriefs in Physics, https://doi.org/10.1007/978-3-030-56168-0

In the original version of the book, author-provided belated corrections have been incorporated as follows:

In Chap. 1, corrections have been incorporated in the third paragraph of Page 1 and in Figs. 1.4, 1.5 and 1.6.

In Chaps. 6 and 7, punctuation has been added to some of the equations.

In Chap. 9 corrections have been incorporated in Fig. 9.5.

In Chap. 10, corrections have been incorporated in the fifth paragraph of Page 69.

In Chap. 11, spacing issue in Equation (11.23) has been corrected.

In Chap. 12, spacing issue in Equations (12.7) and (12.9) and content in the fourth paragraph of Page 96 have been corrected.

In Chap. 13, content in the first paragraph of Page 114 has been corrected.

In Chap. 14, spacing issues in Equations (14.17) and (14.18) have been corrected.

In Chap. 15, the content in the last paragraph in Exercise 3 has been corrected.

Numbers have been added to all the references. The book and the chapters have been updated with the changes.

The updated version of the book can be found at 10.1007/978-3-030-56168-0

(C) The Author(s), under exclusive license to Springer Nature Switzerland AG 2021 